Journal of Case Reports 2017;7(1):89-91

\title{
Perforation of Jejunal Diverticulosis: Rare Cause of Peritonitis
}

\author{
Amandeep Saharan, Satish Dalal, Mahavir Singh, Chisel Bhatia \\ Department of General Surgery, Pt. B. D. Sharma Postgraduate Institute of Medical Sciences (P.G.I.M.S.), Rohtak-124001, Haryana, \\ India.
}

\author{
Corresponding Author: \\ Dr. Chisel Bhatia \\ Email: chiselbhatia@gmail.com \\ This is an Open Access article distributed \\ under the terms of the Creative Commons \\ Attribution License (creativecommons.org/ \\ licenses/by/3.0). \\ Received \\ Accepted \\ December 16, 2016 \\ Published \\ February 1,2017 \\ March 10, 2017
}

\begin{abstract}
Background: Diverticulum of jejunum is a rare entity. Usually it is asymptomatic or may present as chronic abdominal pain, hemorrhage, malabsorption or diverticulitis. Perforation of jejunal diverticulum is difficult to diagnose due to localized peritonitis and requires urgent surgical intervention. Case Report: We hereby report a 52 year old male with perforated jejunal diverticula. Resection of involved gut with end to end jejuno-jejunal anastomosis was done. Conclusion: Peritonitits secondary to perforation of jejunal diverticulum requires high index of suspicion and can be life threatening.
\end{abstract}

Keywords: Abdominal Pain, Diverticulitis, Diverticulum, Jejunum, Peritonitis.

\section{Introduction}

Jejunal diverticula are rare with $1 \%$ prevalence in the general population [1]. Small bowel diverticula occur most frequently in the duodenum (45\%), followed by Meckel's diverticulum (23\%). Jejunal diverticula are the least common type of small bowel diverticula [2]. Jejunal diverticula are usually multiple and predominantly localized to the proximal jejunum, followed by the distal jejunum. They are pseudo-diverticula that only contain mucosa and submucosa [3]. They are beleived to develop as a result of abnormalities in peristalsis, intestinal dyskinesia, and high segmental intraluminal pressures. Use of corticosteroids and non-steroidal anti-inflammatory drugs might play a causative role [4]. Jejunal diverticula are usually asymptomatic but may present as chronic abdominal pain, hemorrhage, malabsorption or can lead to acute complications like diverticulitis, intestinal obstruction and perforation [5].

\section{Case Report}

We report a case of 52-year-old male, who presented in the emergency with upper abdominal pain, vomiting and dyspepsia since last two days. On general physical examination, he had tachypnea, tachycardia with a pulse rate of 96 per minute and his blood pressure was within normal range. Abdominal examination showed tenderness and guarding limited to upper abdomen. Routine laboratory investigations were within normal limits. Abdominal X-rays were normal and there was no air under the right dome of diaphragm. On ultrasound minimal intergut fluid was seen and rest of the findings were normal.

Contrast enhanced CT scan of abdomen showed focal outpouching of contrast from jejunal loop with surrounding fluid and free air foci and thickening of gut loops in the area of right lumber region with possibility of a sealed perforation. On laparotomy, two jejunal diverticula were present on the mesenteric side one foot apart from each other. A perforation measuring $0.5 \times 0.5 \mathrm{~cm}$ was found in the proximal diverticulum which was approximately one foot distal to the duodeno-jejunal junction. Intergut adhesions were present with adhesions between gut and omentum and inflammation of gut limited to proximal jejunum. Peritoneal lavage 
with resection of gut segment containing both the diverticula with end to end anastomosis was done. Postoperative course was uneventful.

\section{Discussion}

Jejunal diverticula are pseudo-diverticula which were first described by Somerling in 1794 and by Sir Astley Cooper in 1807. Their incidence on enteroclysis is $2.0-2.3 \%$ and on autopsy it is 0.06 $4.6 \%$ [1]. Duodenal diverticula are approximately five times more common than jejuno-ileal diverticula. The prevalence increases with age and the disease presents a peak incidence at sixth and seventh decades with a male predominance [6]. They are always situated on the mesenteric border where mesenteric vessels penetrate and are weak. Perforation of jejunal diverticula is a rare complication. The precipitating factors causing perforation have been reported as necrotizing inflammatory reaction, blunt trauma to the abdominal wall and foreign body impaction in a diverticulum [7].

Peritonitis caused by perforated jejunal diverticula can be localized and self-limiting because the diverticula are at mesenteric border of the bowel and readily allow the small bowel mesentery to wall them off. Free air under the diaphragm may not always be visible on chest $\mathrm{X}$ - ray. Although barium scan of the small intestine is one of the best radiological contrast studies to visualize diverticula, it is contraindicated in cases of perforation or acute diverticulitis to suggest a perforation. Abdominal CT scans have been found to be helpful in the diagnosis of jejunal perforation [8].

The treatment of choice for perforated jejunal diverticulum causing peritonitis is exploratory laparotomy and resection of affected intestinal segment with primary anastomosis. The extent of bowel resection depends upon the length of the bowel that is affected by the diverticula and the patient's peri-operative condition [9]. If the diverticula are extensive, resection may have to

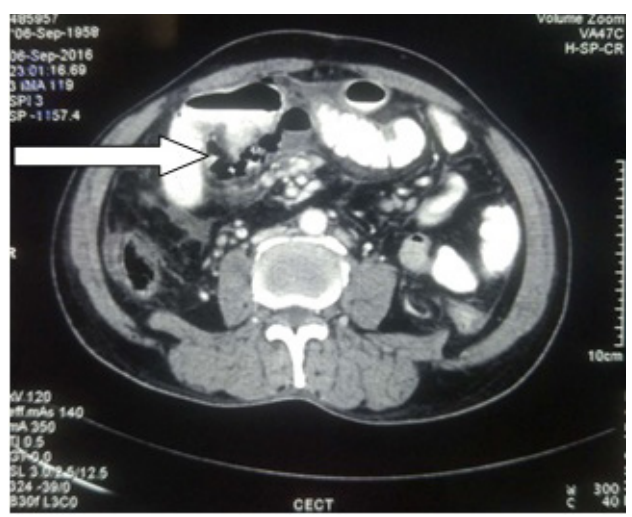

Fig.1: CECT film showing free air around jejunal loops (white arrow).

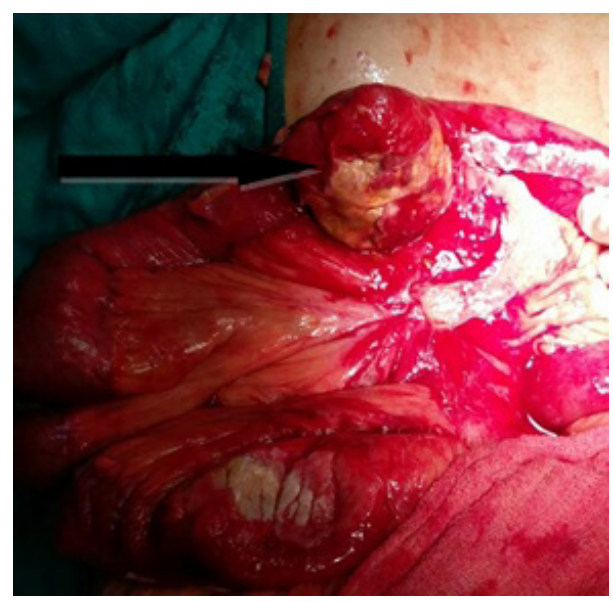

Fig.2: Operative pictures showing perforated jejunal diverticulum (black arrow).

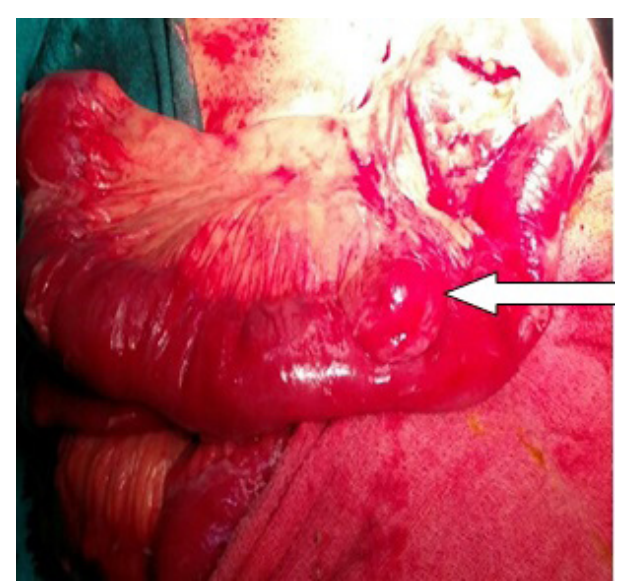

Fig.3: Operative pictures showing intact diverticulum (white arrow). 
be limited to include only the segment containing the perforated diverticulum and to leave a segment of small bowel that still contains non-perforated diverticula in order to avoid short bowel syndrome [10]. Other procedures like simple closure, excision are associated with approximately three times greater mortality rate. Simple diverticulectomy may impair blood flow because of its mesenteric location therefore may lead to anastomotic breakdown or fistula formation. The reported overall mortality rate is $24 \%$, with a mortality rate of $14 \%$ in cases where resection of the involved segment with primary anastomosis was done [11]. The high mortality appears to be related to the advanced age of the patients as well as to delayed diagnosis and treatment.

\section{Conclusion}

Perforation of jejunal diverticula is a rare occurrence. It is difficult to diagnose because of the localized peritonitis it causes. Considering the high mortality associated with this condition, early diagnosis using appropriate investigations like CECT abdomen is the key and surgery with resection of diseased segment with anastomosis is the treatment of choice.

Contributors: AS: Report conception and initial manuscript drafting; SD: Critical review of manuscript; MS, CB: Manuscript revision. All authors were involved in case management. CB will act as guarantor. All authors approved the final version of the manuscript.

Funding: None; Competing interests: None stated.

\section{References}

1. Makris K, Tsiotos GG, Stafyla V, Sakorafas GH. Small intestinal nonmeckelian diverticulosis: clinical review. J Clin Gastroenterol. 2009;43:201-207.

2. Chiu EJ, Shyr YM, Su CH, Wu CW, Lui WY. Diverticular disease of the small bowel. J Hepatogastroenterol. 2000;47:181-184.

3. Butler JS, Collins CG, McEntee GP. Perforated jejunal diverticula: a case report. J Med Case Rep. 2010;4:172.

4. Ozdemir A, Ulas M, Karaman K, Teke Z, Ozer I, Ercan $\mathrm{M}$, et al. Acute abdomen caused by a jejunal diverticulum perforation: A rare clinical entity. Anatol J Clin Investig. 2010;4:168-170.

5. Woods K, Williams E, Melvin W, Sharp K. Acquired jejunoileal diverticulosis and its complications: A review of the literature. Am Surg. 2008;74:849-854.

6. Lempinen M, Salmela K, Kemppainen E. Jejunal diverticulosis: a potentially dangerous entity. Scand J Gastroenterol. 2004;39:905-909.

7. Herrington JL Jr. Perforation of acquired diverticula of the jejunum and ileum: Analysis of reported cases. Surgery. 1962;51:426-433.

8. Fintelmann F, Levine MS, Rubesin SE. Jejunal diverticulosis: Findings on $\mathrm{CT}$ in 28 patients. Am J Roentgenol. 2008;190:1286-1290.

9. Matteoni R, Lolli E, Barbieri A, D’Ambrosi M. Perforated jejunal diverticulitis: personal experience and diagnostic with therapeutical considerations. Ann Ital Chir. 2000;71:95-98.

10. Alvarez J, Dolph J, Shetty J, Marjani M. Recurrent rupture of jejunal diverticula. Conn Med. 1982;46:376378.

11. Roses DF, Gouge TH, Scher KS, Ranson JH. Perforated diverticula of the jejunum and ileum. Am J Surg. 1976;132:649-652. 